\title{
Modeling Broadcasting using Omnidirectional and Directional Antenna in Delay Tolerant Networks as an Epidemic Dynamics
}

\author{
Fernando Peruani*, Abyayananda Maiti ${ }^{\dagger}$, Sanjib Sadhu ${ }^{\dagger}$, Hugues Chaté *, Romit Roy Choudhury ${ }^{\ddagger}$, \\ and Niloy Ganguly ${ }^{\dagger}$ \\ *Service de Physique de l'Etat Condensé (SPEC/CEA) \\ Email: ferperuani@gmail.com and hugues.chate@cea.fr \\ ${ }^{\dagger}$ Dept. of Computer Science and Engineering, IIT Kharagpur, India \\ Email: abyaym,sanjibs,niloy@cse.iitkgp.ernet.in \\ ${ }^{\ddagger}$ Dept. of Electrical \& Computer Engg and Dept. of Computer Sc., Duke University \\ Email: romit@ee.duke.edu
}

\begin{abstract}
We study broadcasting of information in a system of moving agents equipped with omnidirectional as well as directional antenna. The agent communication protocol is inspired by the classical SIRS epidemics dynamics. We assume that the antennas of all agents have a fixed transmitting power, while signal reception only occurs when the receivers sense signals with power exceeding a certain threshold. Thus, information exchange is a local phenomenon which depends on the relative distance and antenna orientation between the transmitting and the receiving agent. We derive an expression for the mean broadcasting time and study the information dissemination robustness of the system using elements of classical epidemiology and physics. In particular, we show that the mean broadcasting time depends upon $\psi$ which quantifies the area the radiation pattern of the antenna sweeps as it moves. We report three important observations (a) directional antennas perform better than omnidirectional antennas, (b) directional antennas whose beam-width is narrower perform even better, and (c) the performance enhances a lot if directional antennas rotate. These behaviors can be understood in the light of the reported analytical findings.
\end{abstract}

Index Terms-Delay tolerant networks, Broadcasting in networks, Epidemic dynamics, SIRS model, Mean field equation, Directional antenna.

\section{INTRODUCTION}

The wide-scale adoption of wireless devices may enable a new platform for peer-to-peer opportunistic networking. One potential framework, termed delay tolerant networks (DTNs) [1], is receiving increasing research attention. Delay tolerant networks comprise of mobile wireless devices that are not necessarily connected to each other (e.g., moving vehicles in a road). Messages progress from one node to another by opportunistically exploiting wireless connectivity as well as physical node mobility. Intermediate nodes receive batches of packets, store and carry them in their local memory, and forward them to other nodes until some (unicast/multicast/broadcast) conditions are satisfied. Examples of

Manuscript received March 03, 2009; revised October 20, 2009. This work was partially supported by the DIT, Govt. of India project grant no. 0527/T/IITKARG/014/0809/33 and STIC-Asie project titled "Information spreading in a system of mobile agents", from the French Government.
DTN application include networking using buses having predictable routes [2], interplanetary networking [3], interfacing with sensor networks [4], and using mobile nodes to bridge data between remote village networks and the Internet [5], [6].

Here, we study the broadcasting properties of a system of moving agents equipped with short-range communication antennas. We show that the broadcasting dynamics of the system depend on agent density, the motility pattern exhibited by the agents, type of antenna, and very importantly, antenna dynamics. We have used specifically two types of antennas: directional antenna and omnidirectional antenna and we refer them as "DA" and "OA", respectively, in rest of the paper. We consider, for simplicity, that there is only one message that is broadcasted and assume a simple transmission dynamics.

In analogy to classical epidemic modeling [7]-[9], agents can be in one of three possible states: susceptible, meaning the agent does not transmit the message, infected, indicating the agent is in broadcasting mode and transmitting the message for a given time (denoted by $\tau_{I}$ ), and recovered, which represents an idle mode of the agent after the broadcasting mode. The recovered, idle state particularly help the energy-strapped mobile agents in conserving energy. The recovered agents after some given time, when the mobile agents have reached an hitherto unexplored region, move back to susceptible state, following the classical SIRS dynamics of epidemiology [7]. A susceptible agent can only switch to broadcasting mode, i.e., infected state, upon reception of the message from a neighbor node.

Broadcasting of a message in DTN with OA has been studied in the past. These previous works can be broadly classified into two categories. (a) A number of approaches have been taken to reduce the overhead and improve the performance of epidemic routing by implementing probabilistic forwarding [10]-[12], redundancy suppression scheme [13] as well as schemes which utilize the history [14]. (b) There have been several works which mainly through extensive simulation have shown the performance of broadcast under different choices of parameters (e.g. message holding time, transmission range, etc) $[15]-[17]$. 


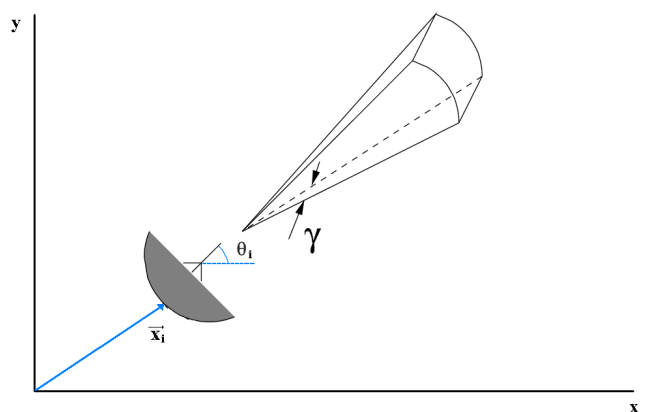

Fig. 1. The state of the $i$-th agent at time $t$ is characterized by its position $\mathbf{x}_{i}$, the orientation of its antenna $\theta_{i}$, and its transmitting mode that can be either $S, I$ or $R$. The agent antenna is characterized by its beamwidth given by $\gamma$.

Here, we analyze a SIRS broadcasting protocol, and derive various performance evaluation metrics, and expressions for the mean broadcasting time and broadcasting robustness of the system, for OA and DA antenna types. One of our main theoretical contributions is to show that the mean broadcasting time is a function of the effective scattering cross section $\psi$, i.e., the new area explored by the agent antenna per unit time, where $\psi$, in turn, is a function of the agent mobility pattern and, very importantly, antenna orientation dynamics. We base our study in previous works on disease spreading on moving agent systems [17]-[20], particularly on [21]. We argue in terms of a simple mean-field theory which, we show, provides a reasonable description of the information spreading dynamics for certain range of parameters. We validate our analytical findings via extensive agent-based stochastic simulations.

Though there are some recent works on information broadcast with DA [22], [23] in static networks, to the best of our knowledge this is the first paper which explores the impact DA produces in context of moving agents with antenna dynamics. The study of DA yields interesting results. We find that DA with same power performs much better than omnidirectional antenna (OA). Interestingly, we find that when the transmission range of DA is comparable to the system size, the performance enhancement cannot be explained only through mean-field theory.

The paper is organized as follows. In section II we introduce an agent-based model. A coarse-grain (analytical) description of such model is derived in section III. Results and conclusions are presented in sections IV and V, respectively.

\section{AGENT-BASED MODEL}

\section{A. Agent motion and antenna orientation dynamics}

We assume agents are self-propelled and move at constant speed in a two-dimensional box with periodic boundary condition, changing their direction of motion at Poissonian distributed times. The equation of motion of the $i$-th agent can be expressed as:

$$
\begin{aligned}
\dot{\mathbf{x}}_{i}(t) & =v \check{V}\left(\alpha_{i}\right) \\
\theta_{i}(t) & =F_{\theta}(t)
\end{aligned}
$$

where $\mathbf{x}_{i}(t)$ represents the position of the $i$-th agent, $\theta_{i}(t)$ denotes the orientation of its antenna (a relevant variable for

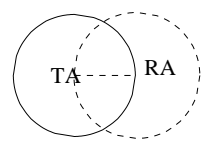

(a)

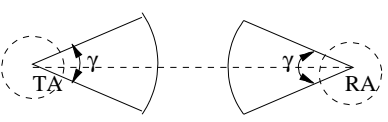

(b)

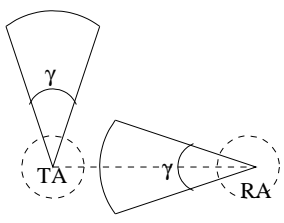

(c)

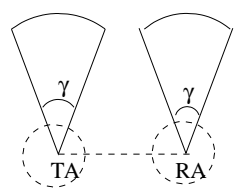

(d)

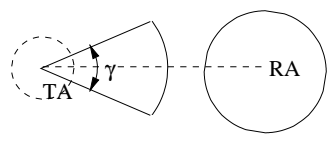

(e)

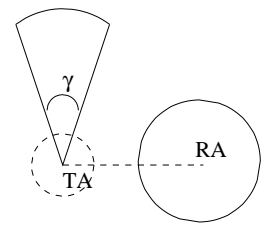

(f)
Fig. 2. Relevant configurations between transmitting antenna (TA) and receiving antenna (RA), depending on the antenna type. Figure (a) corresponds to a pair of OAs. Figures (b), (c), and (d) illustrate the relevant configurations for a pair of DAs. The mixed case, i.e., the configurations corresponding to $\mathrm{OA}$ and DA are illustrated in figures (e) and (f). Notice that if the TA becomes RA and vice versa, the communication range remains the same.

DA, but irrelevant for OA), $v$ is the agent active velocity, and $\check{V}\left(\alpha_{i}\right)$ represents the direction of motion, defined as $\check{V}\left(\alpha_{i}\right)=\cos \left(\alpha_{i}\right) \check{x}+\sin \left(\alpha_{i}\right) \check{y}$. The active direction of motion, determined by the angle $\alpha_{i}$, follows a Poisson process by which an agent changes the direction, i.e., $\alpha_{i}$, at every time step with probability $p_{\text {turn }}$. Eq. (1) defines a continuum time random walk characterized by a diffusion constant $D=$ $v^{2} \kappa^{-1}$, where $\kappa$ represents the angular diffusion experienced by $\alpha_{i}$. For more details see [24]. Assuming that values of $\alpha_{i}$ are strung from a step function of width $\eta$, we can express $\kappa$ as $\kappa=\left(\eta^{2} / 24\right) \cdot p_{\text {turn }}$ [24].

We have also implemented a Poisson dynamics for the orientation of the antennas. This is encoded in the functional form of $F_{\theta}(t)$. Only for DA, the dynamics of its orientation is relevant. The antennas change their orientation at every time step with probability $p_{\text {rot }}$. When $p_{\text {rot }}=0$, the orientation of the antenna never changes. On the other hand, for $p_{\text {rot }}=1$, the antenna orientation changes at every time step.

Thus, the state of an agent is characterized by its position, the orientation of its antenna, and its transmitting mode, that can be either $S, I$ or $R$, as explained below. Moreover, the agent antenna is characterized by its beamwidth, i.e., $\gamma$. See fig. 1 .

\section{B. Signal transmission - the agent antenna}

Imagine that at time $t$ we have the agent $i$ at position $\mathbf{x}_{i}(t)$, whose antenna is pointing in direction $\theta_{i}(t)$, and agent $j$ at position $\mathbf{x}_{j}(t)$ with its antenna pointing in direction $\theta_{j}(t)$. We want to know whether agent $i$ is able to receive a signal sent by agent $j$. This is going to happen only if the $i$ captures a signal sent by $j$ with a power above a certain threshold $\delta$. For 
that we use the Friis transmission formula [25] which can be expressed in two-dimensions as:

$$
P_{r}\left(\mathbf{x}_{i}, \theta_{i}, \mathbf{x}_{j}, \theta_{j}\right)=\frac{K \cdot P_{t}}{\mathbf{x}_{i j}^{2}} G_{T}\left(\check{\theta_{j}} \cdot \mathbf{x}_{i j}\right) G_{R}\left(\check{\theta}_{i} \cdot \mathbf{x}_{j i}\right)>\delta,
$$

where $P_{t}$ is the transimission power of the transmitter, $G_{T}\left(\check{\theta}_{j} \cdot \mathbf{x}_{i j}\right)$ denotes the gain of the transmitter in the direction to the receiver, $G_{R}\left(\check{\theta}_{i} \cdot \mathbf{x}_{j i}\right)$ represents the gain of the receiver in direction of the transmitter, and $\check{\theta_{j}}=\cos \left(\theta_{j}\right) \check{x}+\sin \left(\theta_{j}\right) \check{y}$, while $\mathbf{x}_{i j}=\mathbf{x}_{i}-\mathbf{x}_{j}$.

The functional forms of $G_{T}$ and $G_{R}$ depend on the specific antenna used by the transmitter and receiver, respectively. For more details regarding the Friis transmission formula and the possible functional forms of $G_{T}$ and $G_{R}$ we refer the reader to [25]. For our purposes, we can take these expressions to be:

$$
G_{T / R}\left(\theta_{j}, \mathbf{x}_{j}, \mathbf{x}_{i}\right)= \begin{cases}G_{0}^{M}\left(\gamma^{T / R}\right) & \check{\theta_{j}} \cdot \mathbf{x}_{i j}<\cos \left(\gamma^{T / R} / 2\right) \\ G_{0}^{S} & \check{\theta_{j}} \cdot \mathbf{x}_{i j}>\cos \left(\gamma^{T / R} / 2\right)\end{cases}
$$

where $G_{T / R}$ refers to the transmission and receiving gain, respectively, and $\gamma^{T / R}$ is the angle for maximum gain for the transmitter and receiver. The gain of a DA antenna is defined, at a given distance $d$, as the area of the sphere of radius $d$ divided by area of the solid angle $\gamma$ at distance $d$, assuming that the ideal DA radiates all its power through that solid angle. In a more realistic scenario, one has to assume that antennas exhibit some small leakage of power, which we have modeled by $G_{0}^{S}$. Although there variations among individual DAs, $G_{0}^{S}$ tentatively represents average behavior and is taken as 0.0316 . Here, $G_{0}^{M}$ takes the form: $G_{0}^{M}=1.4 /\left(1-\cos \left(\gamma^{T / R} / 2\right)\right)$ considering 30\% leakage. For more details see [26].

To gain some intuition, we illustrate in Fig. 2 some representative situations. Depending on the type of the antennas and their relative orientations, the maximum transmission radius can be estimated. This is given in table I for the examples shown in Fig. 2. The entries in the table are generated as follow. For omni-directional antennas, gains $\left(G_{T}, G_{R}\right)$ are considered as one and the maximum interaction range $\left(r\left(\mathbf{x}_{i j}\right)\right)$ for omni-omni configuration is also considered to be one. When we consider directional antenna, to maintain fairness, we assume it has same transmission power $P_{t}$, then the maximum interaction range $\left(r\left(\mathbf{x}_{i j}\right)\right)$ is simply determined by the square root of $G_{T} \cdot G_{R}$. For example, take the case of $r_{2}$ for $\gamma=10$. Here, $G_{T}=0.0316$ and $G_{R}=368$, from the formula of $G_{0}^{M}$, then $r_{2}=3.41$.

\section{Broadcasting algorithm}

In this subsection we summarize the steps involved in the implemented broadcasting algorithm, and list the model parameter values. Since computer implementations involve always a discrete time, here we provide for clarity a discrete time description of the algorithm. Our time step is $\Delta t$, a small quantity. The individual-based model works as follows:

Motion of the agents : In each $\Delta t$ we first update the position of the agents. This is done by discretizing Eq. (1) by a simple
TABLE I

RANGES OF DA FOR DIFFERENT $\gamma$ WHERE $r_{1}, r_{2}, r_{3}, r_{4}, r_{5}$ ARE RANGES FOR ANTENNA ORIENTATION OF FIGS. 2( $b, c, d, e, f)$ RESPECTIVELY. HERE THE ASSUMPTION IS THAT BOTH THE SENDING AND RECEIVING ANTENNA HAVE THE SAME $\gamma$.

\begin{tabular}{|l|l|l|l|l|l|}
\hline$\gamma$ (in degree) & $\mathrm{r} 1$ & $\mathrm{r} 2$ & $\mathrm{r} 3$ & $\mathrm{r} 4$ & $\mathrm{r} 5$ \\
\hline \hline 10 & 368 & 3.41 & 0.0316 & 19.2 & 0.18 \\
\hline 15 & 163 & 2.27 & 0.0316 & 12.76 & 0.18 \\
\hline 30 & 41 & 1.14 & 0.0316 & 6.4 & 0.18 \\
\hline 45 & 18 & 0.76 & 0.0316 & 4.24 & 0.18 \\
\hline 60 & 10 & 0.562 & 0.0316 & 3.16 & 0.18 \\
\hline 90 & 5 & 0.4 & 0.0316 & 2.24 & 0.18 \\
\hline
\end{tabular}

Euler method such that $\mathbf{x}_{i}(t+\Delta t)=\mathbf{x}_{i}(t)+v \check{V}\left(\alpha_{i}(t)\right) \Delta t$. Since $\alpha_{i}(t)$ follows a Poisson process, at each time step we decide with probability $p_{\text {turn }}$ whether $\alpha_{i}(t+\Delta t)$ is a new random angle between 0 and $2 \pi$. Otherwise, $\alpha_{i}(t+\Delta t)=$ $\alpha_{i}(t)$. Initial direction of motion of each agent, $\alpha_{i}(0)$, is taken as random.

Antenna Direction : We update the orientation of the antenna according to (2) (for OA this step is irrelevant). Similarly to what we do with $\alpha_{i}(t)$, since $\theta_{i}(t)$ also follows a Poisson process, we decide at each time step with probability $p_{\text {rot }}$ whether $\theta_{i}(t+\Delta t)$ is a new random angle between 0 and $2 \pi$ [27]. Otherwise, $\theta_{i}(t+\Delta t)=\theta_{i}(t)$. The initial direction of antenna of each agent, i.e., $\theta_{i}(t=0)$, is taken as random.

Message State : Here we update the message state of each agent. If the $i$-th agent is in state "S", we explore the space looking for all $j$-agents in the system in state "I", that is, transmitting the message, and checking whether there is at least one of them that fulfills Eq. (3). We replace the state of the $i$-th agent by "I" if an agent is found. On the other hand, if the $i$-th agent is in either "I" or " $\mathrm{R}$ " state, we advance the internal clock associated with that state by $\Delta t$. If the state is "I" and the internal clock marks a time larger than $\tau_{I}$, we change the state of the agent to " $R$ " and reset the clock. If the state is " $\mathrm{R}$ " and the clock indicates a time larger than $\tau_{R}$, the agent moves back to the "S" mode. The transition from I to $\mathrm{R}$, and from $\mathrm{R}$ to $\mathrm{S}$ is a fully deterministic process. Initially only a single agent is in state "I".

The table II indicates the model parameters. Those parameter values shown in the table have been kept fixed across the paper. Though the number of agents $N$ has been varied, most of the simulations were performed with $N=1000$ agents. The linear size of the system is indicated by $L$. The density $\rho$ is defined as $\rho=N / L^{2}$, assuming rectangular area.

\section{MEAN-FIELD APPROACH}

When communication occurs most frequently between pairs of agents (i.e, three or more agents talking simultaneously is a rare event), the agent spatial distribution is roughly homogeneous, and the system is well-mixed, we can expect a mean-field approach to provide a reasonable description of the information dynamics. However, it is well-known that a mean-field description for systems as the ones we deal with in this paper cannot provide an accurate description of the system dynamics either close to its phase transition point or at 
TABLE II

MOdEl PARAMETERS (SEE TEXT FOR DETAILS).

\begin{tabular}{|l|l|}
\hline Parameter & value \\
\hline \hline $\mathrm{N}$ & varied (1000 in most cases) \\
\hline $\mathrm{L}$ & varied \\
\hline $\mathrm{v}$ & 0.1 \\
\hline$p_{\text {turn }}$ & varied $(0.0001$ in most cases) \\
\hline$p_{\text {rot }}$ & varied \\
\hline$\gamma$ & varied \\
\hline$G_{0}^{S}$ & 0.0316 \\
\hline$\tau_{I}$ & 500 \\
\hline$\tau_{R}$ & 50 \\
\hline
\end{tabular}

very high densities (see for instance [21], [28] and references therein). The mean-field time evolution of the populations of susceptible, infected, and recovered agents is described by the following equations:

$$
\begin{aligned}
\dot{S} & =\frac{R}{\tau_{R}}-(\rho \psi) I S \\
\dot{I} & =(\rho \psi) I S-\frac{I}{\tau_{I}} \\
\dot{R} & =\frac{I}{\tau_{I}}-\frac{R}{\tau_{R}}
\end{aligned}
$$

where $S, I$ and $R$ are defined as $S=N_{S} / N, I=N_{I} / N$ and $R=N_{R} / N$, with $N$ being the total number of agents in the system, and $N_{S}, N_{I}$, and $N_{R}$ being the number of susceptible, infected and recovered agents, respectively. The product $(\rho \psi) S$ describes the mean number of susceptible agents an infected agent infects per unit time, where $\rho$ stands for the agent density and $\psi$ represents the (new) area an agent explores per time unit [21]. For a system with only OA agents, $\psi$ can be expressed as $\psi=v \sigma_{0}$, where $v$ is the agent speed and $\sigma_{0}$ the effective scattering cross section, a classical concept of kinetic gas theory [29]. For a system with only OAs with the same $P_{t}$ for all antennas, $\sigma_{0}$ takes the simple form $\sigma_{0}=2 r$, where $r$ represents maximum interaction range of the antenna. For the used parameters, $r=1$. For systems with DA agents, or mixed systems, we can assume that $\psi$ can be approximated by

$$
\psi=\left(a(\gamma) p_{\text {rot }}\right)+(v \sigma(\gamma)),
$$

where $a(\gamma)$ represents the new area DA explores each time on changing its orientation, which is function of the antenna beam width $\gamma$, while $\sigma(\gamma)$ denotes the effective scattering cross section of the DA.

\section{A. Robustness condition}

By taking the LHS of Eqs. (5)-(7) equal to zero, we obtain the steady states of the system, which are: i) $S=1, I=0$, and $R=0$, corresponding to the epidemic extinction, i.e., no agent transmitting the message, and ii)

$$
\begin{aligned}
S_{s t} & =R_{0}^{-1} \\
I_{s t} & =\left(1-S_{s t}\right) \frac{\tau_{I}}{\tau_{I}+\tau_{R}} \\
R_{s t} & =\left(1-S_{s t}\right) \frac{\tau_{R}}{\tau_{I}+\tau_{R}},
\end{aligned}
$$

corresponding to the endemic state, where $R_{0}=\psi \rho \tau_{I}$ corresponds in epidemic dynamics to the so-called basic reproductive number [21]. Eqs. (9)-(11) define a system state where there is always a finite fraction of agents transmitting the message, i.e., $I(t \rightarrow \infty)>0$. Strictly speaking, this is only true for infinite systems.

Linearizing Eqs. (5)-(7) around the steady states given by i) and ii), it is easy to see that the stability of the steady states changes at $R_{0}=1$. The mean-field approximation provides us with a condition for the existence of a non-vanishing asymptotic fraction of transmitting agents which reads:

$$
\psi \rho \tau_{I}>1 .
$$

For a given $\psi$ and $\rho$, we can use condition (12) to estimate $\tau_{I}$, i.e., the time period agents should retransmit the message to avoid message broadcast extinction. Clearly, if we can assure that a finite fraction of agent will be always broadcasting the message, we can also assure that asymptotically everybody is going to receive the message. In that way, condition (12) defines the broadcast robustness condition of the system.

\section{B. Average broadcasting time}

Now we want to estimate the average broadcasting time. For that, we keep track in simulations of the number of individual agents that have received the message since the beginning of the numerical experiment. We define $Y(t)$ as the fraction of informed individuals at time $t$, i.e., the number of agents that have received the message at least once since the beginning of the experiment. Thus, $1-Y(t)$ is the fraction that still has not received the message. We can express the time evolution of $Y(t)$ as:

$$
\dot{Y}=\psi \rho(1-Y) I
$$

The reasoning behind Eq. (13) is that each of the $I(t)$ agents that are broadcasting the message will transmit the message (per unit time) to $\psi \rho(1-Y)$ agents that have not still received the message. If we assume that we know $I(t)$, we can think of Eq. (13) as a simple non-homogeneous ordinary differential equation whose solution is:

$$
Y(t)=\left(\frac{1}{N}-1\right) \exp \left[-(\psi \rho) \int_{0}^{t} d t^{\prime} I\left(t^{\prime}\right)\right]+1,
$$

where it has been assumed that at $t=0$ there is only one agent informed and transmitting the message. A similar expression to Eq. (14) has been derived in [20] in the context of SI and SIR message broadcasting dynamics.

One can define the broadcasting time as the time at which all individuals in the system have received the message. We refer to this time as $T_{b}$. However, according to Eq. (14), that condition is fulfilled when $Y(t)=1$. Mathematically speaking, this occurs for $t \rightarrow \infty$. No doubt that in a finite system, in simulations for example, $Y(t)=1$ for a finite time $t$. Although this is true, Eq. (14) suggests that such a definition of $T_{b}$ leads to values strongly dominated by fluctuations. To overcome these problems, we propose to use an alternative 


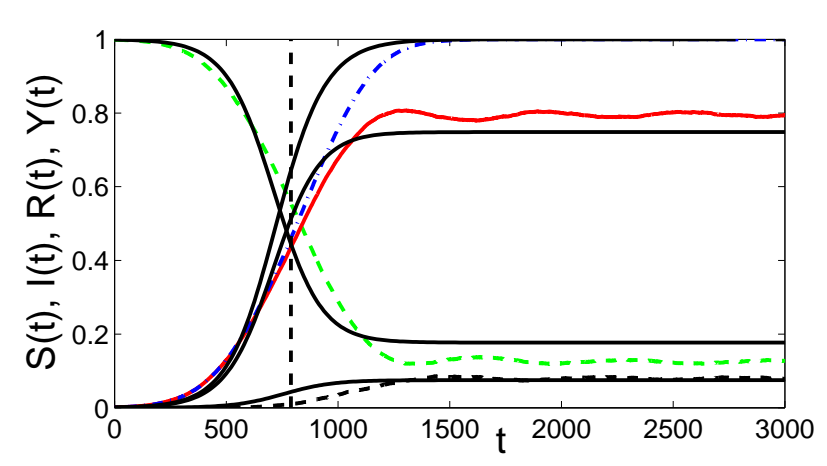

Fig. 3. Time evolution of $S$ (green dashed), $I$ (red solid), $R$ (black dashed), and $Y$ (blue dash-dotted) for a system with $N=1000$ agents with $\mathrm{OA}$ at a density $\rho=0.06$. Black solid curves correspond to the mean-field approach, integration of Eqs. (9)-(11) and (14). The vertical black dashed line corresponds to Eq. (15).

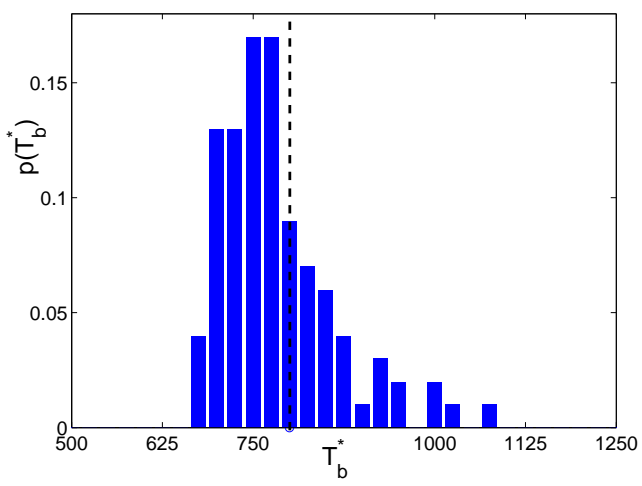

Fig. 4. Histogram of the broadcasting time $T_{b}^{*}$ (based on 100 realizations), i.e., Eq. (15), for a system with $N=1000$ agents with OAs at a density $\rho=0.06$. Black dotted line denotes the average broadcasting time.

definition for broadcasting time. According to Eq.(14), $Y(t)$ experiences a crossover when:

$$
\int_{0}^{T_{b}^{*}} d t^{\prime} I\left(t^{\prime}\right)=\frac{1}{\psi \rho}
$$

where $T_{b}^{*}$ is the new defined broadcasting time. Notice that the exponential in Eq. (14) defines, as usual, a characteristic time. Similarly we can express this condition by asking when $Y(t)=1-\exp (-1)$, which occurs at $t=T_{b}^{*}$.

\section{RESUlTS}

\section{A. Omnidirectional antenna}

We start by studying the case where all agents are equipped with identical OAs. At intermediate densities, the mean-field approach provides, as mentioned above, a reasonable description of the information spreading dynamics. Both, transient and steady state behavior are well described. Fig. 3 compares the mean-field approach and simulations. Notice that the mean-field approach does not involve any fitting parameters. From the condition given by Eq. (12), the critical density $\rho_{c}=1 /\left(\psi \tau_{I}\right)$, above which the message can be successfully broadcasted, can be derived. Recall that for OA, $\psi=v \sigma_{0}$. Thus, $\rho_{c}=0.01$.

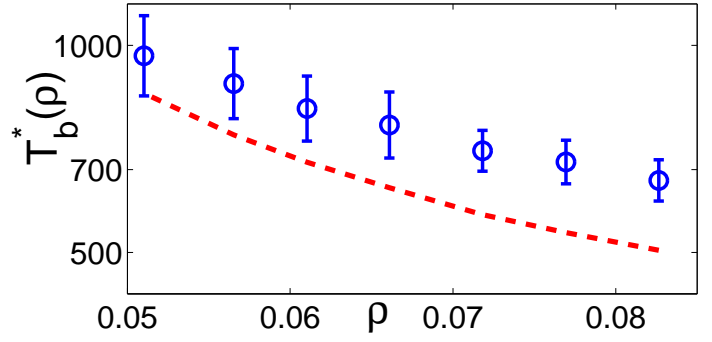

Fig. 5. Average broadcasting time $T_{b}^{*}$ as function of the agent density $\rho$. Simulations were performed using $N=1000$ agents. Each circle corresponds to the average of 100 simulations, and vertical bars indicate the standard deviation. The red dashed curve indicates the theoretical prediction given by Eq. (15). Notice that there is no fitting parameter.

In the following, we focus on the mean broadcasting time. Fig. 4 shows the histogram obtained for the mean broadcasting time $T_{b}^{*}$, for a density $\rho=0.06$. The histogram is based on 100 realizations of the same numerical experiment. Interestingly, the distribution of $T_{b}^{*}$ is not a symmetric Gaussian distribution. The vertical dashed line correspond to the average $T_{b}^{*}$. A more systematic study of the nature of the distribution $P\left(T_{b}^{*}\right)$ is left for future research.

Finally, we show that Eq. (15) can be used to predict $T_{b}^{*}$ at different densities. Fig. 5 shows $T_{b}^{*}$ as function of the agent density $\rho$. Each circle corresponds to an average over 100 simulations, while the dotted curve is the prediction given by Eq. (15). As it can be observed, the mean-field approach provides a reasonable description of the broadcasting dynamics of the system at intermediate densities without any fitting parameter.

\section{B. Omnidirectional vs. directional antenna}

In this subsection we study the effect of DA usage in the broadcasting time $T_{b}^{*}$. In simulations, DA agents have the same values of $K P_{t}$, and $\delta$ as OA agents. Recall that the maximum interaction range between the two agents depends on the value of $\gamma^{T}$ of each of the agents. Fig. 6 shows that the broadcasting time $T_{b}^{*}$ decreases as the fraction of DA agents $\left(\rho_{D A}\right)$ is increased, where $\rho_{D A}=N_{D A} / N$, with $N_{D A}$ the number of agents equipped with DA antenna. Notice that for $\rho_{D A}=0$, i.e., for only OA agents, $T_{b}^{*}$, as expected, does not depend on $p_{\text {rot }}$. The figure also shows that as the rotation probability $p_{\text {rot }}$ increases, $T_{b}^{*}$ decreases at least initially at faster pace. When the system entirely comprises of directional antenna, i.e, for $\rho_{D A}=1$, we observe even when $p_{\text {rot }}=0$ that the system performs always better than OA irrespective of the antenna angle.

Fig. 7 focuses on the two extreme cases, $p_{\text {rot }}=0$ (a) and $p_{\text {rot }}=1$ (b). When $p_{\text {rot }}=0$, the evolution of message broadcasting depends exclusively on agent migration, i.e., $\psi=(v \sigma(\gamma))$. Thus, Fig. 7 (a) shows in an indirect way the behavior of the effective scattering cross section $\sigma(\gamma)$ for a mixture of $\mathrm{OA}$ and $\mathrm{DA}$, characterized by a $\rho_{D A}$ (detailed discussed later). On the other hand, when $p_{\text {rot }}=1$, the new area explored by the antenna per unit time is dominated by the turnings performed by the antenna direction, i.e., 


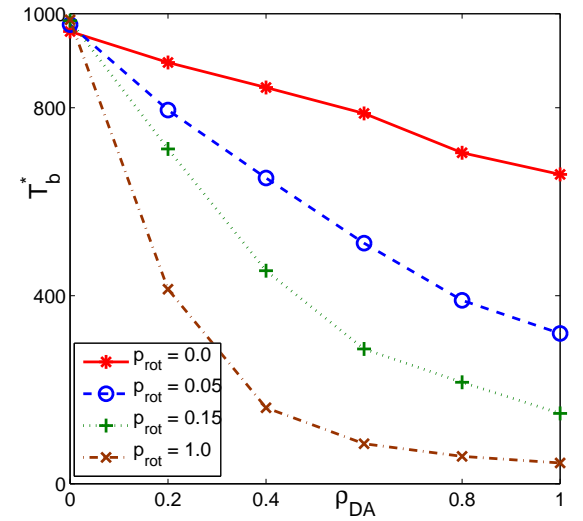

Fig. 6. Average broadcasting time $T_{b}^{*}$ vs. DA fraction $\rho_{D A}$ for various rotation probability $p_{\text {rot }}$, agent density $\rho=0.05$, beam width $\gamma=60$. (a)

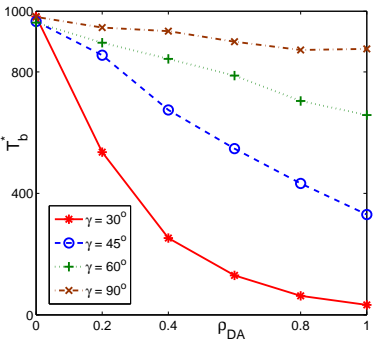

(b)

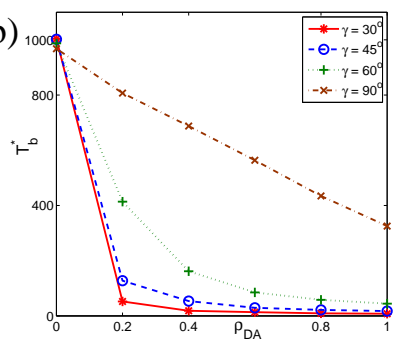

Fig. 7. Average broadcasting time $T_{b}^{*}$ vs. DA fraction $\rho_{D A}$ for various values of antenna beam width $\gamma$ with rotation probability $p_{\text {rot }}=0$ (a) and $p_{\text {rot }}=1$ (b) for an agent density $\rho=0.05$.

$\psi=\left(a(\gamma) p_{\text {rot }}\right)$. As it can be seen in Fig. 7 (b), these effects become more pronounced for smaller values of $\gamma$. Another important point which can be observed in both Figs. 7 (a) and (b) is that the steep decrease in broadcasting time $T_{b}^{*}$ for small values of $\rho_{D A}$ indicates that the introduction of a small number of DA in the system can dramatically improve the broadcasting performance.

Fig. 8 shows the response of $T_{b}^{*}$ as agent density $\rho$ is changed. The simulation data indicates that DA performs better for all densities. The figure also shows that $T_{b}^{*}$ monotonically decreases, for all agent density $\rho$, as the fraction of DA agents, $\rho_{D A}$, is increased. Inset plot of Fig. 8 describes the comparative performance of a system with all DA agents over the system with all OA agents. It shows that in higher density DA system performs better. This also highlights the benefits of the longer range transmission of DA agents in higher density where it broadcasts the message more quickly by reaching to more number of distant agents.

Initial infected agent count : Another interesting aspect could be to analyze results for varying number of initially infected agents. This would require understanding the interaction among the agents which can be complex and we believe would need a much more detailed look. However, from initial experimentation we have noticed some interesting results which we are reporting here. As expected, it is seen uniformly the broadcasting time decreases with increasing number of initial

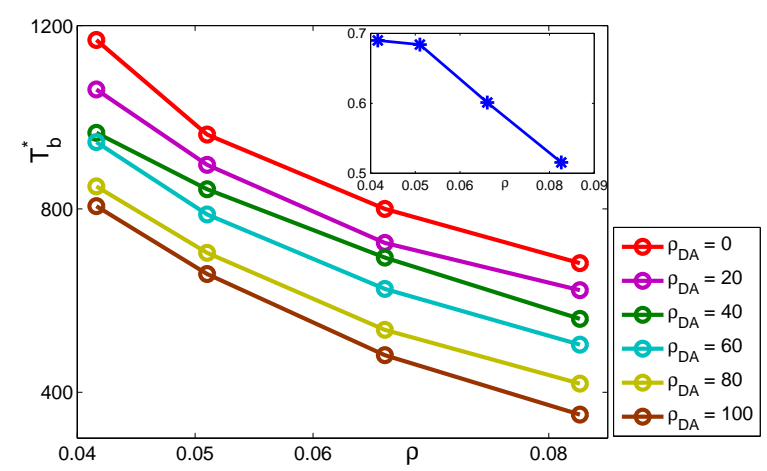

Fig. 8. Average broadcasting time $T_{b}^{*}$ vs. agent density $\rho$ for various values of DA fraction $\rho_{D A}$, for $\gamma=60, p_{\text {rot }}=0$. Inset figure shows the Ratio of $T_{b}^{*}$ of system with $\rho_{D A}=100$ and $\rho_{D A}=0$ for different agent density.

agents. However, it is observed that with an increasing fraction of DA, the effect of multiple initial agents is better again supporting the fact that the DA can distribute the infectious agents to remote places whereby each can explore an area on its own.

\section{Antenna and agent rotation}

Here we study the impact of $p_{\text {turn }}$ to the broadcasting performance. We also study its relation with antenna rotation $p_{\text {rot }}$, specifically, by considering three situations. (a) The antenna does not turn even though its corresponding agent has turned. (b) The antenna's direction is fixed with respect to the direction of the agent, and in consequence if the agent turns, for example, 10 degrees to the West, the antenna will also turn 10 degrees to the West. (c) The antenna turns independent of the agent's direction. Fig 9 explains all the above three situations.

In order to understand the sole effect of agent rotation, we consider, at first, only omni-directional antenna. We find that the broadcasting time increases as agents turning probability increases (red lines on both Figs. 9 (a) and (b) which correspond to $\rho_{D A}=0$ ). With increasing rotation probability $p_{t u r n}$, each individual agent moves around within a small area, thus losing the chance of exploring new area and coming in contact with new potential agents. This same feature is noticed in case of DA where $T_{b}^{*}$ steadily increases when the DA does not change direction (black and blue circle-line in Fig 9 (a)). However, if antenna is rotated exactly the amount the agent moves, $T_{b}^{*}$ decreases with $p_{\text {turn }}$ if the number of DA antennas is large enough, as the curve for $\rho_{D A}=20$ and $p_{\text {rot }}=0$ (blue circle-line) in Fig 9(b) indicates. For smaller values of $\rho_{D A}$, the broadcasting performance may not improve by increasing agent rotation, since for agents with OA, large values of $p_{\text {turn }}$ imply larger values of $T_{b}^{*}$ (see curve for $\rho_{D A}=0$ ). Nevertheless, for intermediate values of $\rho_{D A}$ a decrease in $T_{b}^{*}$ can be observed at large values of $p_{t u r n}$ (black circle-line in Fig 9(b)). When the antenna moves independently of the direction of agents, as can be expected, motion hardly has any impact. We also show the situation where the random rotation of the antenna is done after the antenna gets aligned 

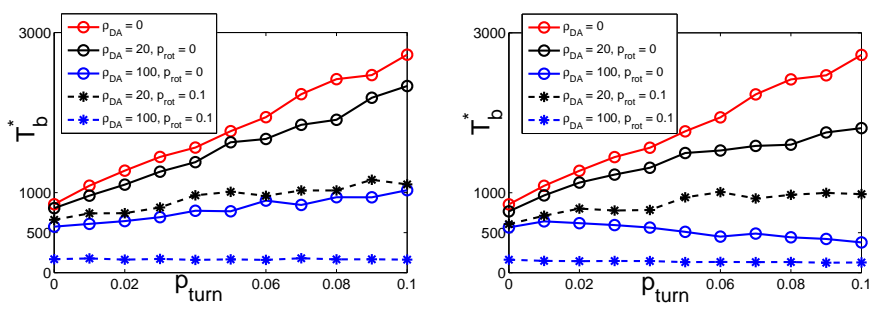

Fig. 9. Average broadcasting time $T_{b}^{*}$ vs. probability to change the node's moving direction $p_{\text {turn }}$ for various values of DA fraction $\rho_{D A}$ and antenna direction change probability $p_{\text {rot }}(\gamma=60$ in case of DA nodes). (a) change of antenna direction is independent of node's direction, (b) node's direction change is reflected in antenna direction change. The same curve for $\rho_{D A}=$ 0 (only omni-directional) is drawn in both (a) \& (b) to maintain parity

with the agents motion (black and blue star-line Fig 9 (b)). The broadcasting time is exactly same if the rotation is done without performing the alignment (black and blue star-line Fig 9 (a)). All these findings can be explained by studying the behavior of $\psi$.

\section{Analysis of Results}

From the above stated detailed simulation results we can infer mainly four important observations:

1) Systems with DA agents with $p_{r o t}=1$ demonstrates lower broadcast time than systems of DA agents with $p_{\text {rot }}=0$.

2) DA agents with smaller $\gamma$ perform (in term of $T_{b}^{*}$ ) comparatively better than the agents with larger $\gamma$. This is true for all values of $p_{\text {rot }}$.

3) Independent of other parameters, system with DA agents always show better results compared to the system of only OA agents $\left(\rho_{D A}=0\right)$.

4) The performance of DA agents becomes better if it is aligned with the direction of the agent motion.

As explained in the previous paragraphs, a closer look at the mean field equations (Eqs. (5)-(7)) suggests that the possible way of understanding these findings is by thinking in terms of $\psi$ (Eq. (8)). The reasons behind the first and fourth observations are already given earlier in that line. To explain the second observation for $p_{\text {rot }}=0$ we have to calculate the value of $\psi$ hence the value of $v \sigma(\gamma)$ for DAs.

Calculation of $\psi$ for DA agents is little involved and depending on the receiving agents and relative orientation of antennas etc. Hence here due to shortage of space only a representative table showing the $\psi$ 's calculated for different $\gamma$, considering a simplified situation that movement in all direction is equiprobable (Table III). The table immediately confirms the reason behind the better performance of DAs with smaller $\gamma$ (observation 2). About the third observation, we have seen that in all cases systems with DA agents perform better than the OA agents. From the mean field approach (Eqs. (5)-(7)) we know that $T_{b}^{*}$ depends on the value of $\psi$. So apparent reason for better performance of DA agents should be its larger $\psi$. But the table shows that $\psi$ of DAs with larger $\gamma$ is very close to $\psi$ of $\mathrm{OA}$ (which is 0.2 ) which points to the fact that $\psi$ cannot fully explain the reason behind better performance of DA over OA. This may be due to the reason
TABLE III

VALUES EXPECTED $\psi$ FOR DIFFERENT VALUES OF $\gamma$ FOR DA ANTENNA WITH $v=0.1$

\begin{tabular}{|l|l|l|l|l|l|}
\hline$\gamma($ in degree $)$ & 30 & 45 & 60 & 90 & Omni \\
\hline$E(\psi)$ & 0.3138 & 0.2374 & 0.1976 & 0.1769 & 0.2 \\
\hline
\end{tabular}

that DA agents are creating some random long links and the message is quickly distributed to remote areas. In other words, DA induces a collapse of the diameter of the system. Under these circumstances, the mean field equations fail, providing a too large overestimated broadcasting time $T_{b}^{*}$. As the system size increases, mean field predictions become better.

\section{CONCLusions}

This paper makes a systematic and analytic study to understand and establish the potential of DA in DTN. We have systematically introduced Directional Antenna in an epidemic setting, used the classical Friis equation to model message transmission, derived a mean-field, based on the agent mobility and antenna dynamics, to describe information broadcasting dynamics, and then performed extensive simulation to understand the behavior of DA. Two important insights have been developed. First, DA direction can be suitably changed to attain a larger new explored area per time unit $(\psi)$, and hence faster information dissemination can be achieved. Secondly, narrow but long-range antenna can substantially enhance the speed of broadcasting. The affectivity and versatility of the DA can be further established considering more realistic agent mobility settings, some examples of which are found in [30][32]. However, the two above mentioned realizations will help in suitably couple DA with specific mobility pattern to build efficient information dissemination algorithms.

\section{ACKNOWLEDGMENT}

The authors would like to extend their gratitude to Subrata Nandi of IIT Kharagpur, India for his valuable comments and suggestions.

\section{REFERENCES}

[1] "Delay tolerant network research group(dtnrg) http://www.dtnrg.org."

[2] K. Fall, "A delay-tolerant network architecture for challenged internets," in ACM SIGCOMM, 2003.

[3] S. Burleigh, A. Hooke, L. Torgerson, K. Fall, V. Cerf, B. Durst, and K. Scott, "Delay tolerant networking: An approach to interplanetary internet," IEEE Communication Magazine, vol. 41, no. 6, pp. 128-136, 2003.

[4] A. Mainwaring, J. Polastre, R. Szewczyk, D. Culler, and J. Anderson, "Wireless sensor networks for habitat monitoring," in ACM International Workshop on Wireless Sensor Networks and Applications(WSNA Ó2), Atlanta, GA, 2002.

[5] S. Jain, K. Fall, and R. Patra, "Routing in a delay tolerant networks," in ACM/SIGCOMM, Oregon, USA, Aug 30-Sept 32004.

[6] A. Pentland, R. Fletcher, and A. Hasson, "Daknet: Rethinking connectivity in developping nations," IEEE Computer, vol. 37, pp. 128-136, January 2004.

[7] J. D. Murray, Mathematical Biology, 3rd ed., ser. Mathematics Book Club (U.S.A.) adoption. Heidelberg: Springer-Verlag, 1993.

[8] O. Diekmann and J. A. P. Heesterbeek, Mathematical Epidemiology of Infectious Diseases: Model Building, Analysis and Interpretation, ser. Mathematical and computational biology. Wiley, 2000. 
[9] Z. Ma and J. Li, Basic Knowledge and Developing Tendencies in epidemic dynamics, ser. Book: Mathematics for Life Science and Medicine. Springer, 2007.

[10] T. Matsuda and T. Takine, "(p,q )-epidemic routing for sparsely populated mobile ad hoc networks," IEEE JOURNAL ON SELECTED AREAS IN COMMUNICATIONS, vol. 26, no. 5, JUNE 2008.

[11] X. Zhang, G. Neglia, J. Kurose, and D. Towsley, "Performance modeling of epidemic routing," in IFIP Networking, 2006

[12] Y. C. Tseng, S. Y. Ni, Y. S. Chen, and J. P. Sheu, "The broadcast storm problem in a mobile ad hoc network," Wireless Networks, vol. 8, no. $2 / 3,2002$.

[13] T. Small and Z. Haas, "Resource and performance tradeoffs in delay tolerant wireless networks," in ACM SIGCOMM Workshop on Delay Tolerant Networking (WDTN), 2005.

[14] P. Juang, H. Oki, Y.Wang, M. Martonosi, L. S. Peh, and D. Rubenstein, "Energy-efficient computing for wildlife tracking: Design tradeoffs and early experiences with zebranet," in ACM ASPLOS, 2002.

[15] Z. Zhang, "Routing in intermittently connected mobile ad hoc networks and delay tolerant networks: overview and challenges," IEEE Соттиnications, vol. 8, no. 1, 2006.

[16] A. Vahdat and D. Becker, "Epidemic routing for partially connected ad hoc networks, technical report cs-200006," Duke University, Tech. Rep., April 2000.

[17] D. M. Zhang, "Mean-field equations and stable behaviour in an epidemic model of mobile individuals," Phys. Scr.,pp. 73-78, 2006.

[18] N. Boccara and K. Cheong, "Critical behaviour of a probabilistic automata network sis model for the study of an infectious disease in a populations of moving individuals," J. Phys. A: Math. Gen., vol. 26, pp. 3707-3717, 1993.

[19] M. C. Gonzalez, P. G. Lind, and H. J. Herrmann, "System of mobile agents to model social networks," Phys. Rev. Lett., vol. 96, p. 088702, 2006.

[20] E. Altman, T. Basar, and F. de Pellegrini, "Optimal monotone forwarding policies in delay tolerant mobile ad-hoc networks," in Inter-Perf 2008: Workshop on Interdisciplinary Systems Approach in Performance Evaluation and Design of Computer Communication Systems, Athens, Greece, October 2008.

[21] F. Peruani and G. Sibona, "Dynamics and steady states in excitable mobile agent system," Phys. Rev. Lett., vol. 100, p. 168103, 2008.

[22] M. Chuah and Y. Xi, "Enhanced delivery in disruption tolerant networks using advantageous nodes with directional antenna capability," in Milcom, 2007.

[23] C. Shen, Z. Huang, and C. Jaikaeo, "Directional broadcast for mobile ad hoc networks with percolation theory," IEEE Transactions on Mobile Computing, vol. 5, pp. 317-332, April 2006.

[24] F. Peruani and L. Morelli, "Self-propelled particles with fluctuating speed and direction of motion in two dimensions," Phys. Rev. Lett., vol. 99, p. 010602, 2007.

[25] J.D.Kraus, Antennas, 2nd ed. McGraw-Hill, 1988.

[26] R. Ramanathan, "On the performance of ad hoc networks with beamforming antennas," in International Symposium on Mobile Ad Hoc Networking \& Computing(MobiHoc 01), Long Beach, CA, USA, October 2001.

[27] R. Choudhury and N. Vaidya, "Impact of directional antennas on ad hoc routing," in Personal and Wireless Comm. (PWC), 2003.

[28] R. Dickman and D. S. Maia, "The nature of the absorbing-state phase transition in the diffusive epidemic process," J. Phys. A: Math. Theor. vol. 41, p. 405002, 2008.

[29] F. Reif, Fundamentals of Statistical and Thermal Physics. McGrawHill, Singapore, 1985

[30] M. González, C. Hidalgo, and A.-L. Barabási, "Understanding individual human mobility patterns," Nature, vol. 453, pp. 479-482, 2008.

[31] D. Brockmann, L. Hufnagel, and T. Geisel, "The scaling laws of human travel," Nature, vol. 439, pp. 462-465, 2006.

[32] T. Vicsek, A. Czirók, E. Ben-Jacob, I. Cohen, and O. Shochet, "Novel type of phase transition in a system of self-driven particles," Phys. Rev. Lett., vol. 75, no. 6, pp. 1226-1229, August 1995.

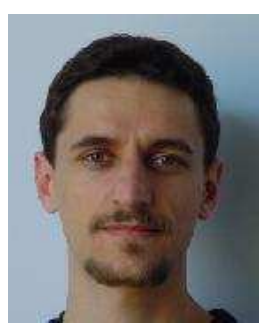

Fernando Peruani is a CEA post-doc at the Service de Physique de l'Etat Condese, France, since 2008. He received a Bachelor and Master degree in Physics from the University of Buenos Aires, Argentina (2003). He then moved to the Max Planck for the Physics of Complex Systems, Germany, from where he got a $\mathrm{PhD}$ degree (2007). His research interests include non-equilibrium statistical physics, active matter, complex networks, and information spreading in mobile ad hoc networks.

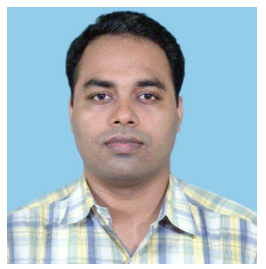

Abyayananda Maiti received his B. Tech. degree in Computer Science and Engineering from Haldia Institute of Technology, India in 2001. He is now pursuing his M.S. degree in Computer Sc and Engineering from IIT Kharagpur, India. His research interests include modeling complex network growth and delay tolerant networks.

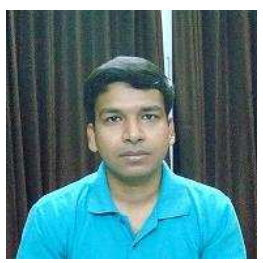

Sanjib Sadhu received his B.E. degree in Computer Science and Engineering from Regional Engineering College Durgapur, India in 2001 and the M.Tech degree in CSE from National Institute of Technology (NIT), Durgapur, India in 2006. He is now a faculty member in CSE at NIT, Durgapur. His research interests include delay tolerant networks and image processing.

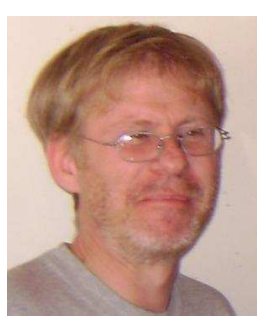

Hugues Chaté is a senior scientist at the Condensed Matter Physics department of CEA-Saclay, France. A graduate from Ecole Centrale Paris, he received his $\mathrm{PhD}$ in theoretical physics from Universit Pierre et Marie Curie in Paris in 1989. After a brief postdoc at Bell Labs, he joined CEA-Saclay, where he has been conducting research on non-equilibrium and nonlinear physics, with a recent focus on active matter issues, and in particular on the collective properties of self-propelled particles.

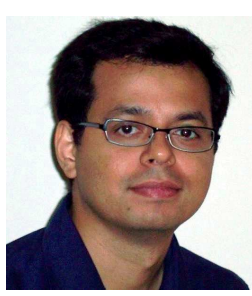

Romit Roy Choudhury is an Assistant Professor of ECE and CS at Duke University. He joined Duke in Fall 2006, after completing his PhD from UIUC. His research interests are in wireless protocol design mainly at the PHY/MAC layer, and in distributed mobile computing at the application layer. He received the NSF CAREER Award in January 2008. Visit Romit's Systems Networking Research Group (SyNRG), at http://synrg.ee.duke.edu.

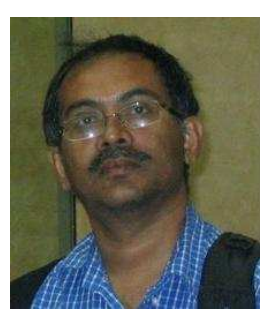

Niloy Ganguly is an Associate Professor in the Department of Computer Science and Engineering, Indian Institute of Technology, Kharagpur. He has received his B.Tech from IIT Kharagpur in 1992 and his $\mathrm{PhD}$ from BESU, Kolkata, India in 2004. He has spent two years as Post-Doctoral Fellow in Technical University, Dresden, Germany before joining IIT, Kharagpur in 2005. His research interests are in peer-to-peer networks in particular and distributed, dynamic networks in general. He has applied various bio-inspired techniques to solve problems in such networks. He also works on applying complex networks methodology in various information retrieval problems. He is presently leading a research group comprising of several research scholars and masters student as well as various collaborators from industry and academia. Visit the Complex Networks Research Group (CNERG) at www.cse-web.iitkgp.ernet.in/ cnerg/. 